\title{
On Stability of Open-Loop Operation without Rotor Information for Brushless DC Motors
}

\author{
Zhong Wu, ${ }^{1}$ Haotun Lyu, ${ }^{1}$ Yongli Shi, ${ }^{2}$ and Di Shi ${ }^{1}$ \\ ${ }^{1}$ School of Instrumentation Science and Optoelectronics Engineering, Beihang University, Beijing 100191, China \\ ${ }^{2}$ Beijing Institute of Control Engineering, Chinese Academy of Space Technology, Beijing 100190, China
}

Correspondence should be addressed to Zhong Wu; wuzhong@buaa.edu.cn

Received 17 February 2014; Accepted 8 June 2014; Published 1 July 2014

Academic Editor: Alexei Mailybaev

Copyright (c) 2014 Zhong Wu et al. This is an open access article distributed under the Creative Commons Attribution License, which permits unrestricted use, distribution, and reproduction in any medium, provided the original work is properly cited.

Open-loop operation mode is often used to control the Brushless DC Motors (BLDCMs) without rotor position sensors when the back electromotive force (EMF) is too weak due to the very low rotor velocity. The rotor position information is not necessary in this mode and the stator windings are supplied with voltages under a certain ratio of the amplitude to the frequency. However, the rotor synchronization will be destroyed once if the commutation instant is inappropriate. In order to improve the reliability of the open-loop operation mode, a dynamic equation is established to represent the synchronization error between the rotor and the stator. Thereafter, the stability of the open-loop control mode is analyzed by using Lyapunov indirect method. Theoretical analysis indicates that the open-loop control mode is asymptotically stable only when the commutation instant of the stator current lags behind the ideal one suitably. Finally, theoretical analysis is verified through the experimental results of a certain BLDCM.

\section{Introduction}

The Brushless DC Motor (BLDCM) is an AC synchronous motor with a permanent-magnet rotor and can be electronically commutated. Due to their advantages of smaller size, higher efficiency, simpler structure, easier control, and higher performance, BLDCMs have been widely used in many fields $[1,2]$, such as aerospace, military equipment, electrical vehicles, CNC machines, computer peripherals, instruments, and domestic appliances.

In order to realize high-performance control of BLDCMs, the rotor position sensors are often mounted in the motors to obtain the information of the rotor position and velocity. However, the existence of the rotor position sensors will not only increase the size, weight, and the cost but also decrease the reliability of the motor systems. Even if the simple switch-mode hall position sensor is used, the reliability can not be guaranteed in the environment of high temperature, strong vibration, and strong corrosion. Sensorless control of BLDCMs is a feasible solution to the applications in the harsh environment as well as high-reliability concerns $[2,3]$.

Among the various sensorless control strategies for BLDCMs, the back electromotive force (EMF) method has been widely used to detect the rotor position due to its ease of implementation $[4,5]$. However, the effective information of the rotor position is difficult to be derived from the weak back EMF when the motor is standstill or operates at a very low velocity. In such cases, the open-loop operation mode is often used to control the motors. In this mode, the rotor position information is not necessary and the stator windings are supplied with voltages under a certain ratio of the amplitude to the frequency (i.e., $V / f$ ). However, the rotor synchronization will be destroyed by the improper commutation instant resulting from the improper $V / f$. Especially in the BLDCMs of large inertia or heavy load, the stability problem of the open-loop operation mode is quite serious.

Early in the 1980s, the operation stability of the permanent magnet synchronous motors (PMSM) was analyzed theoretically by the authors in $[6,7]$. After that, a method of adjusting the ratio of voltage to frequency was proposed based on the analysis of its effects on the stability of PMSMs in $[8,9]$. Matsui $[10]$ also proposed a method to start the motor according to the information of the winding voltage and current directly. However, these studies only focused on the PMSMs with sinusoidal back EMF and the BLDCMs with trapezoidal back EMF are not involved. 
For the BLDCMs with trapezoidal back EMF, many algorithms were proposed to smooth the starting procedure from standstill to a minimum speed at which the back EMF is large enough to provide the information of the rotor position [11-17]. However, few of them dealt with the problem of operation stability for BLDCMs. Although Shen and Tseng mentioned the relationship between the commutation angle and the operation performance in [4], the detailed theoretical analysis is not available.

The motivation of this paper is to demonstrate the stability condition through theoretical analysis and improve the reliability of the open-loop operation mode for BLDCMs. Hence, a dynamic equation is established to represent the synchronization error between the rotor and the stator at first. Thereafter, the stability of the open-loop operation mode is analyzed by using Lyapunov indirect method. The remaining parts of the paper are organized as follows. In Section 2, a brief description of the principles and stability problem of the open-loop mode for BLDCMs will be given. In Section 3, a dynamic equation representing the synchronization error between the rotor and the stator will be established for BLDCMs, and then the mean electromagnetic torque relying on the commutation angle is derived. In Section 4, the stability of the open-loop operation mode will be analyzed by using Lyapunov indirect method. In Section 5, experimental results of a certain BLDCM drive are presented to verify the stability condition obtained in this paper. Finally, conclusions are made in Section 6.

\section{Problem Description}

2.1. Principles of Open-Loop Operation Mode for BLDCMs. In the sensorless control of BLDCMs based on the detection of the back EMF, the open-loop operation mode is often used to start and accelerate the motor since the back EMF is too weak to provide the information of the rotor position when the motor is standstill or operates at a very low speed. In this mode, the stator windings are energized according to the commutation state machine instead of the actual rotor position, as shown in Figure 1. The commutation state machine driven by a signal with ramp frequency contains 6 fixed states in a certain sequence and the initial one depends on the prepositioning pulse or the predetermined rotor position. The supplied voltage varies with the ramp frequency at a certain ratio, also known as the variablevoltage variable-frequency control. Furthermore, the ratio of $V / f$ should be designed properly according to the motor and the load parameters so that the rotor can be forced to follow the ramp frequency by the enough electromagnetic torque.

However, the rotor is difficult to follow the variation of the stator current rapidly due to its large time constant for the BLDCM with large inertia or heavy load. Once an improper ratio of $V / f$ is used, the rotor will tend to oscillate and even step out due to the improper commutation instant. Thus, a starting failure is incurred. Therefore, the stability should be analyzed theoretically to improve the reliability of the openloop operation mode for BLDCMs.

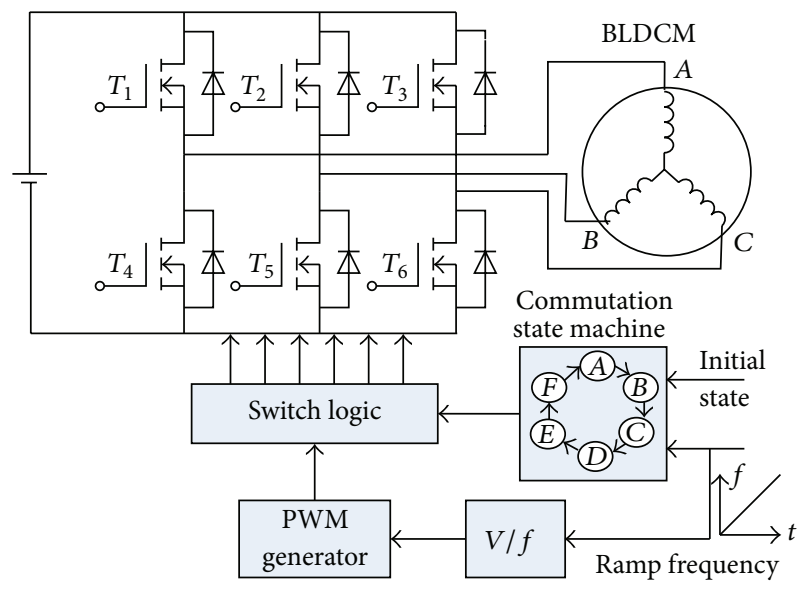

FIGURE 1: Schematics of open-loop operation mode.

2.2. Description of Stability Problem for Open-Loop Operation Mode in BLDCMs. There are three possible phase relationships between the back EMF and the phase current in BLDCMs, as shown in Figure 2. When the commutation angle $\Delta \theta=0$, the phase current is in phase with the back EMF and the commutation instant is accurate. When $\Delta \theta<0$, the phase current leads the back EMF and the commutation instant is advanced. When $\Delta \theta>0$, the phase current lags behind the back EMF and the commutation instant is retarded. For the BLDCMs in the open-loop operation mode without rotor position information, the operation stability may be affected by the sign of the commutation angle $\Delta \theta$.

For convenience, assume that the BLDCM has ideal back EMF of trapezoidal waveform with a $120^{\circ}$ constant plateau and is fed with rectangular stator currents. Let $I, \theta_{s}, \mu_{i}(\cdot)$ denote the amplitude, phase, and the unit waveform function of the stator current, respectively, and then the phase currents can be expressed as

$$
\begin{aligned}
& i_{a}=I \mu_{i}\left(\theta_{s}\right), \\
& i_{b}=I \mu_{i}\left(\theta_{s}-120^{\circ}\right), \\
& i_{c}=I \mu_{i}\left(\theta_{s}+120^{\circ}\right) .
\end{aligned}
$$

Let $\theta_{e}, E, \mu_{e}(\cdot)$ denote the electrical angle of the rotor, the amplitude, and the unit waveform function of the back EMF, respectively, and then the back EMF can be expressed as

$$
\begin{aligned}
& e_{a}=E \mu_{e}\left(\theta_{e}+90^{\circ}\right), \\
& e_{b}=E \mu_{e}\left(\theta_{e}-30^{\circ}\right), \\
& e_{c}=E \mu_{e}\left(\theta_{e}+210^{\circ}\right),
\end{aligned}
$$

where $\left(\theta_{e}+90^{\circ}\right)$ implies that the back EMF leads the rotor flux by $90^{\circ}$.

Let $\omega_{s}=d \theta_{s} / d t$ and $\omega_{e}=d \theta_{e} / d t$ denote the angular frequency of the stator currents and the rotor flux (or the back EMF), respectively, and then the stability problem of the open-loop operation mode for BLDCMs can be described as follows. 


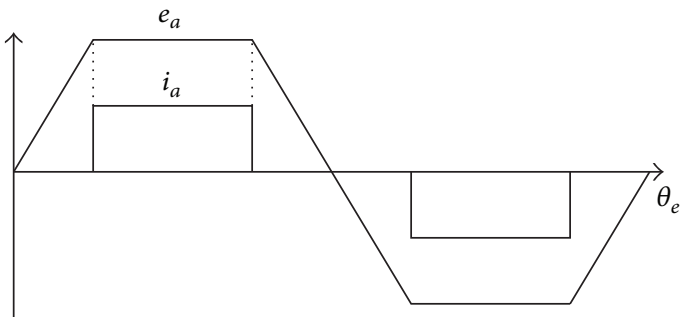

(a) Accurate commutation $(\Delta \theta=0)$

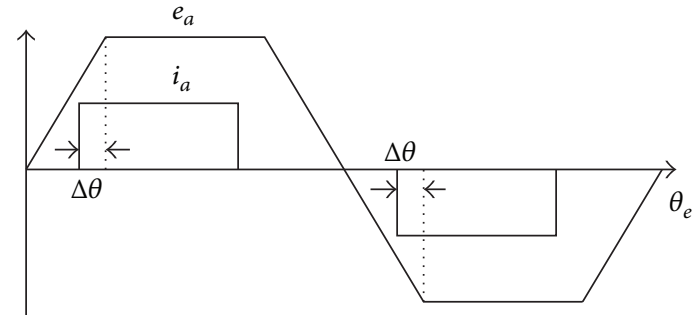

(b) Advanced commutation $(\Delta \theta<0)$

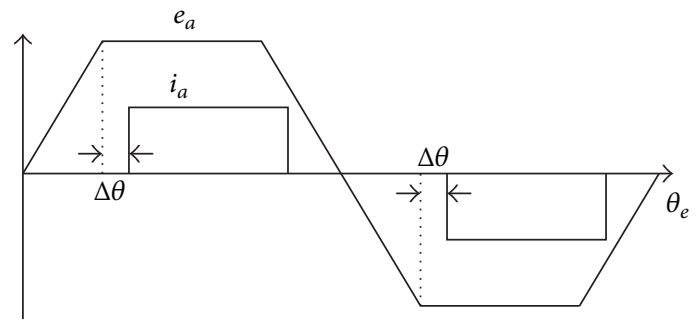

(c) Retarded commutation $(\Delta \theta>0)$

Figure 2: Different commutation conditions.

If both the speed synchronization difference $\Delta \omega=\omega_{e}-\omega_{s}$ and the commutation angle $\Delta \theta=90^{\circ}+\theta_{e}-\theta_{s}$ are bounded asymptotically, the BLDCM will be stable in the open-loop operation mode without the rotor position information.

Obviously, the bounded $\Delta \omega$ ensures the synchronization between the rotor flux (or the back EMF) and the stator currents, while the bounded $\Delta \theta$ ensures the production of the electromagnetic torque large enough to maintain the stable operation of the motor.

\section{Mathematical Models}

3.1. Error Dynamics of Rotor Synchronization. In order to analyze the stability of the BLDCM in the open-loop operation mode, mathematical models should be established to represent the synchronization error between the rotor and the stator. Considering a BLDCM with a surface-mounted permanent-magnet rotor and 3-phase wye-connected symmetric stator windings, neglecting the salient and slot effects, the magnetic saturation, and the losses due to hysteresis and eddy current, then the electromagnetic torque can be written as

$$
T_{e}=\frac{1}{\omega}\left(e_{a} i_{a}+e_{b} i_{b}+e_{c} i_{c}\right),
$$

where $\omega$ is the angular speed of the rotor. According to (1)-(3), it is known that the electromagnetic torque depends on not only the current amplitude but also the phase difference $\Delta \theta$ between the back EMF and the stator currents. The maximum of the electromagnetic torque can be achieved only when $\Delta \theta=0$.

Actuated by the torque in (3), the rotor motion can be described as

$$
J \frac{d \omega}{d t}=T_{e}(\Delta \theta)-T_{L}-D \omega,
$$

where $T_{e}(\Delta \theta)$ denotes the torque function of $\Delta \theta$ and $J, D$, and $T_{L}$ are the moment of inertia, damping coefficient, and the load torque, respectively.

From (4), the dynamic equation representing the synchronization error between the rotor and the stator can be derived as

$$
\begin{aligned}
& \Delta \dot{\theta}=\Delta \omega, \\
& \Delta \dot{\omega}=\frac{p}{J} T_{e}(\Delta \theta)-\frac{D}{J} \Delta \omega-\frac{p}{J} T_{L}-\frac{D}{J} \omega_{s}-\dot{\omega}_{s},
\end{aligned}
$$

where $p$ is the number of pole pairs.

Henceforth, the problem of stability analysis for the BLDCMs in the open-loop operation mode can be transformed into the qualitative study on the nonlinear ordinary differential equation (5).

3.2. Derivation of Mean Electromagnetic Torque Relying on Commutation Angle. In order to analyze the stability of the open-loop operation mode based on (5), the analytical expression of $T_{e}(\Delta \theta)$ should be given first. For the BLDCMs with trapezoidal back EMF, the rotor flux linkages $\psi_{r a}, \psi_{r b}$, and $\psi_{r c}$ can be written as [18-20]

$$
\left[\begin{array}{l}
\psi_{r a} \\
\psi_{r b} \\
\psi_{r c}
\end{array}\right]=\psi_{r m} \sum_{n=1}^{\infty} k_{2 n-1}\left[\begin{array}{c}
\sin \left((2 n-1) \theta_{e}\right) \\
\sin \left((2 n-1)\left(\theta_{e}-120^{\circ}\right)\right) \\
\sin \left((2 n-1)\left(\theta_{e}+120^{\circ}\right)\right)
\end{array}\right],
$$

where $\psi_{r m}$ is the magnitude of the fundamental component of the permanent magnet flux linkage, $k_{n}$ is the coefficient of the $n$th flux harmonic relative to the fundamental, and $k_{1}=1$. For 3 -phase wye-connected symmetric stator windings, the even harmonics, the third harmonic, and its multiples are canceled out in (6). 
Replace the back EMF with the rotor flux linkages in (3), we can get

$$
T_{e}=p\left[\frac{d \psi_{r a}\left(\theta_{e}\right)}{d \theta_{e}} i_{a}+\frac{d \psi_{r b}\left(\theta_{e}\right)}{d \theta_{e}} i_{b}+\frac{d \psi_{r c}\left(\theta_{e}\right)}{d \theta_{e}} i_{c}\right] .
$$

Substitute (6) into (7), and then the electromagnetic torque in presence of back EMF harmonics can be expressed as

$$
T_{e}=\psi_{r m} \sum_{n=1}^{\infty} k_{2 n-1}(2 n-1) \mathbf{i}^{T}\left[\begin{array}{c}
\cos \left((2 n-1) \theta_{e}\right) \\
\cos \left((2 n-1)\left(\theta_{e}-120^{\circ}\right)\right) \\
\cos \left((2 n-1)\left(\theta_{e}+120^{\circ}\right)\right)
\end{array}\right],
$$

where $\mathbf{i}=\left[i_{a}, i_{b}, i_{c}\right]^{T}$.

Since the analytical relationship between $T_{e}$ and $\Delta \theta$ is difficult to be derived from (8) in the 3-phase stationary reference frame, it can be expressed in the synchronously rotating $d-q$ reference frame. After $d-q$ transformation, the fundamental component of flux linkage is transformed into a dc component, while the 5th and the 7th harmonics are transformed into the 6th harmonic, the 11th and the 13th harmonics are transformed into the 12th harmonic, the 17 th and the 19th harmonics are transformed into the 18th harmonic, and so on [19]. Then the electromagnetic torque in $d$-q reference frame can be expressed as [18]

$$
T_{e}=\frac{3 p}{2} \psi_{r m}\left[i_{q}\left(1+h_{d}\right)+i_{d} h_{q}\right],
$$

where $i_{d}, i_{q}$ are stator currents in $d$-q frame, $h_{d}, h_{q}$ are higher-order harmonics of rotor flux in $d$ - $q$ frame, and

$$
\begin{aligned}
& h_{d}=\sum_{n=1}^{\infty}\left[(6 n-1) k_{6 n-1}+(6 n+1) k_{6 n+1}\right] \cos \left(6 n \theta_{e}\right), \\
& h_{q}=\sum_{n=1}^{\infty}\left[(6 n-1) k_{6 n-1}-(6 n+1) k_{6 n+1}\right] \sin \left(6 n \theta_{e}\right) .
\end{aligned}
$$

Owing to the infinite series and rectangular-waveform currents in (9), it is too complicated to find out the analytical expression of the commutation angle $\Delta \theta$. Hereby, the mean values of the rotor flux and the stator currents during a commutation interval can be used to simplify (9).

As shown in Figure 3, the rotor along $d$-axis rotates continuously at a speed of $\omega_{e}$ while the stator current $\mathbf{i}$ steps forward every $60^{\circ}$ with an average speed of $\omega_{s}$. The intersection angle between $q$-axis and $\mathbf{i}$ is $\left(\Delta \theta-30^{\circ}\right)$ at the beginning and $\left(\Delta \theta+30^{\circ}\right)$ at the end of the commutation interval. Let $\gamma$ denote an angle in $\left[-30^{\circ}, 30^{\circ}\right]$, and then the intersection angle can be represented by $(\Delta \theta+\gamma)$ during a certain commutation interval. Thus, the mean values of the stator current $\mathbf{i}$ in the $d$ - and $q$-axes can be derived as

$$
\begin{aligned}
& \bar{i}_{q}=\frac{3}{\pi} \int_{-\pi / 6}^{\pi / 6} I \cos (\Delta \theta+\gamma) d \gamma=\frac{3}{\pi} I \cos \Delta \theta, \\
& \bar{i}_{d}=\frac{3}{\pi} \int_{-\pi / 6}^{\pi / 6} I \sin (\Delta \theta+\gamma) d \gamma=\frac{3}{\pi} I \sin \Delta \theta .
\end{aligned}
$$

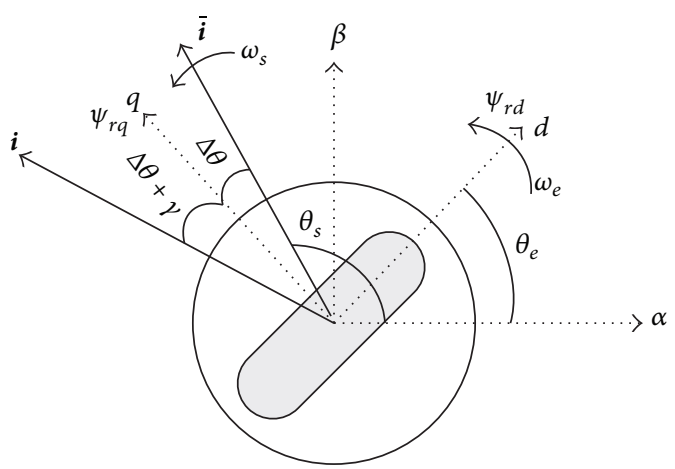

FIgURE 3: Schematics of stator current and rotor flux.

Correspondingly, the mean values of the higher-order harmonics $h_{d}$ and $h_{q}$ during a certain commutation interval also can be derived from (10) as follows:

$$
\begin{aligned}
& \bar{h}_{d}=\frac{3}{\pi} \int_{\theta_{0}}^{\theta_{0}+\pi / 3} h_{d} d \theta_{e}=0, \\
& \bar{h}_{q}=\frac{3}{\pi} \int_{\theta_{0}}^{\theta_{0}+\pi / 3} h_{q} d \theta_{e}=0,
\end{aligned}
$$

where $\theta_{0}$ is the angular position of the rotor at the beginning of the commutation interval.

Replacing $i_{d}, i_{q}, h_{d}, h_{q}$ with their mean values $\bar{i}_{d}, \bar{i}_{q}, \bar{h}_{d}, \bar{h}_{q}$ in (9), then the mean electromagnetic torque can be achieved as

$$
\bar{T}_{e}=\frac{9 p}{2 \pi} \psi_{r m} I \cos \Delta \theta
$$

Obviously, the explicit function of the electromagnetic torque relying on the commutation angle $\Delta \theta$ is given in (13).

\section{Stability of Open-Loop Operation}

If the electromagnetic torque $T_{e}$ is replaced with its mean value $\bar{T}_{e}$, the error dynamics (5) of the open-loop operation mode for BLDCMs can be changed into

$$
\begin{aligned}
& \Delta \dot{\theta}=\Delta \omega, \\
& \Delta \dot{\omega}=\frac{9 p^{2}}{2 \pi J} \psi_{r m} I \cos \Delta \theta-\frac{D}{J} \Delta \omega-\frac{p}{J} T_{L}-\frac{D}{J} \omega_{s}-\dot{\omega}_{s} .
\end{aligned}
$$

Obviously, the error dynamics in (14) is nonlinear. For the stability of nonlinear dynamical systems, various results have been derived in [21-27]. For convenience, the stability of (14) can be analyzed by using Lyapunov indirect method. Linearize (14) at the initial commutation angle $\Delta \theta_{0}$, and then the following linearized error dynamics can be achieved as

$$
\left[\begin{array}{c}
\Delta \dot{\theta} \\
\Delta \dot{\omega}
\end{array}\right]=A\left[\begin{array}{c}
\Delta \theta \\
\Delta \omega
\end{array}\right]-\frac{1}{J}\left[\begin{array}{c}
0 \\
p T_{L}+D \omega_{s}+J \dot{\omega}_{s}
\end{array}\right],
$$

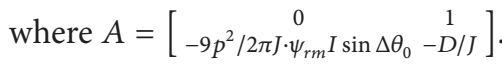


The characteristic equation of the state matrix $A$ can be expressed as

$$
\lambda^{2}+\frac{D}{J} \lambda+\frac{9 p^{2}}{2 \pi} \frac{\psi_{r m} I}{J} \sin \Delta \theta_{0}=0 .
$$

According to Lyapunov indirect method, the conclusions concerning the operation stability of BLDCMs are derived from (16) as follows.

When $\Delta \theta_{0}<0$ (i.e., advanced commutation), system (14) is instable and the motor can not operate in the open-loop mode without rotor position information stably since there is an eigenvalue with positive real part in (16).

When $\Delta \theta_{0}>0$ (i.e., retarded commutation), system (14) is asymptotically stable and the motor can operate in the openloop mode without rotor position information stably since both two eigenvalues of (16) have negative real parts.

When $\Delta \theta_{0}=0$ (i.e., accurate commutation), the stability of system (14) can not be judged from the linearized system (15) since one eigenvalue of (16) is 0 and the other has negative real part. Even if system (14) is marginally stable, its robustness to the disturbances is very poor. Once the commutation angle $\Delta \theta$ turns to be negative owing to certain disturbances, the motor will lose stability and the reliability of the open-loop operation mode can not be guaranteed. In contrast, the BLDCM in the self-control mode (commutated with the rotor information) not only operates stably but also can realize maximal electromagnetic torque, minimal power, and the highest efficiency when $\Delta \theta_{0}=0$.

According to the aforementioned analyses, it is clear that the commutation instant of the stator currents should lag behind the ideal one suitably so as to realize stable operation of the BLDCM in the open-loop mode. In the practical systems, the energized voltage should be large enough to produce enough electromagnetic torque which can drive the rotor advanced.

Compared with the relative studies, the main contribution of this paper is to overcome the difficulties in analyzing the operation stability by using the error dynamics with the mean electromagnetic torque and demonstrate the stability conditions of the open-loop mode for BLDCMs. In particular, it is pointed out that the stability of the open-loop mode is sensitive to the disturbances during the accurate commutation and the reliability can not be guaranteed.

\section{Experimental Results}

In order to verify theoretical analyses in Section 4 , experiments were carried out with a BLDCM. The configuration of the experimental system is shown in Figure 4 and the parameters of the motor and drive system are listed in Table 1.

During the experiment procedure, the rotor of the BLDCM should be aligned to a predetermined position by energizing two phases first, and then it can be accelerated with the open-loop operation mode. In order to examine the effects of different commutation angles on the operation stability of the motor, the initial duty cycle of PWM is chosen as $30 \%$ to force the rotor advanced appropriately by a larger voltage supplied to the stator windings. After that, reduce the
TABLE 1: Motor and drive system parameters.

\begin{tabular}{lc}
\hline Parameters & Values \\
\hline Rated speed & $3000 \mathrm{r} / \mathrm{min}$ \\
Rated voltage & $24 \mathrm{~V}$ \\
Stator winding resistance & $0.9 \Omega$ \\
Stator winding inductance & $0.27 \mathrm{mH}$ \\
Damping coefficient & $4.14 \times 10^{-5} \mathrm{Nm} /{\mathrm{rad} \cdot \mathrm{s}^{-1}}^{-6} \mathrm{Kg} \cdot \mathrm{m}^{2}$ \\
Inertia & $3.8 \times 10^{-6}$ \\
Number of phases & 4 \\
Number of pole pairs & $3-\mathrm{phase} \mathrm{full} \mathrm{bridge}$ \\
Drive circuit & $\mathrm{MOSFET}$ \\
Power switch component & TMS320F28335 \\
Control core & $15 \mathrm{KHz}$ \\
PWM frequency & \\
\hline
\end{tabular}

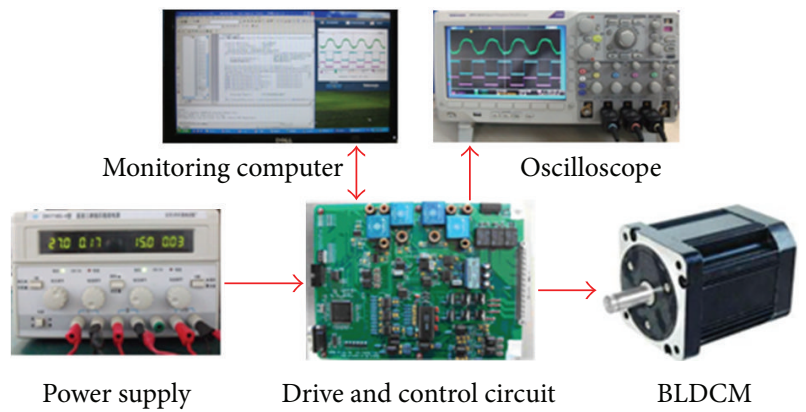

FIgURE 4: Configuration of experimental system.

duty cycle of PWM gradually, and then the supplied voltage to the stator windings will be decreased correspondingly so that the rotor will lag little by little and different phases between the rotor flux (or back EMF) and the stator current will appear.

Following the experimental method stated above, different kinds of commutation phases can be achieved, such as advanced, accurate, and retarded commutation. Experimental waveforms of winding voltage, hall signal, and actual commutation signal for phase A are shown in Figure 5 Figure 8. In each figure, the upper curve is the winding voltage $u_{a}$ detected by a resistance divider and a low-pass filter and the lower one is the hall signal $H_{a}$ whose rising or falling edge represents the ideal commutation instant, while the middle one is the DSP output signal $s_{a}$ whose rising or falling edge represents the actual commutation instant. It should be pointed out that $s_{a}$ displayed on the oscilloscope is an inverted signal due to the experimental setup. Hence, the phase difference between the rising edge of $s_{a}$ and the falling edge of $H_{a}$ represents $\Delta \theta$ actually.

5.1. Advanced Commutation. Figure 5 shows the experimental curves when the actual commutation instant leads the ideal one by $14^{\circ}$ or so. From Figure 5 , it can be seen that the absolute value of the phase difference $\Delta \theta$ has a trend of divergence since it is growing bigger and bigger. Simultaneously, the waveform of the winding voltage deteriorates 


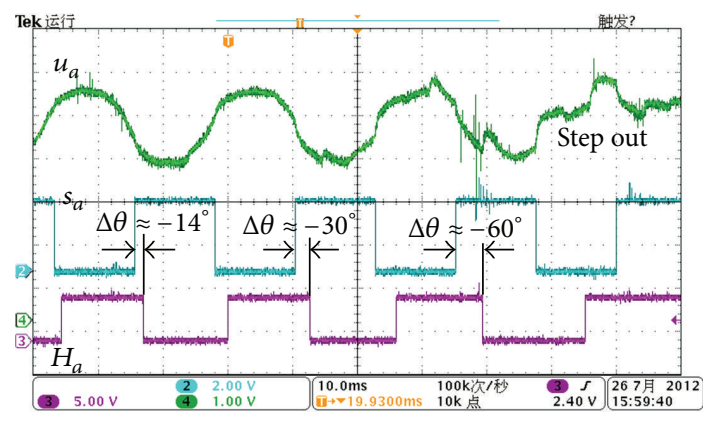

Figure 5: Curves of advanced commutation $\left(\Delta \theta \approx-14^{\circ}\right)$.

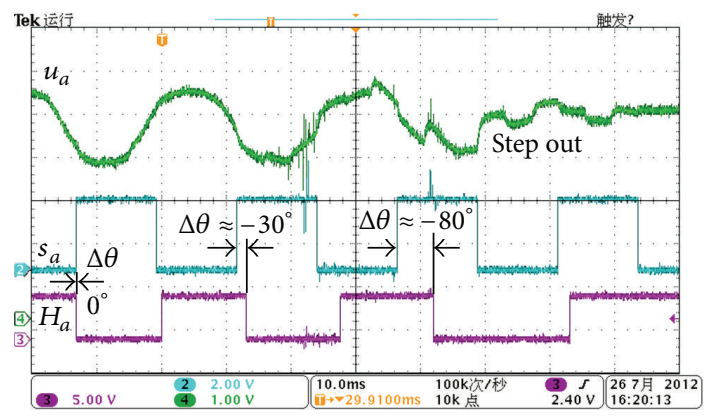

FIGURE 6: Curves of accurate commutation $\left(\Delta \theta \approx 0^{\circ}\right)$.

sharply while the rotor steps out until it stops. It conforms to the theoretical analyses in Section 4; that is, the motor can not operate stably in the open-loop mode when the stator current leads the back EMF.

5.2. Accurate Commutation. Figure 6 shows the experimental curves when the actual commutation instant coincides with the ideal one approximately. From Figure 6, it can be seen that the waveform of the winding voltage deteriorates sharply and the rotor steps out until it stops although the initial phase difference $\Delta \theta_{0}$ between the stator current and the back EMF is about 0 and the commutation instant is accurate. It also conforms to the theoretical analyses in Section 4; that is, the motor will lose stability in the open-loop operation mode when $\Delta \theta_{0}=0$ because the antidisturbance performance of the system is poor.

5.3. Retarded Commutation. Figures 7 and 8 show the experimental curves when the actual commutation instant lags behind the ideal one by $14^{\circ}$ and $30^{\circ}$, respectively. It can be seen that the waveforms of the winding voltage remain similar and the motor can operate stably. This also conforms to the theoretical analyses in Section 4; that is, the motor can operate stably in the open-loop mode when the stator current lags behind the back EMF.

\section{Conclusions}

In order to improve the reliability of the open-loop operation mode, this paper establishes a dynamic equation to represent the synchronization error between the rotor and the stator

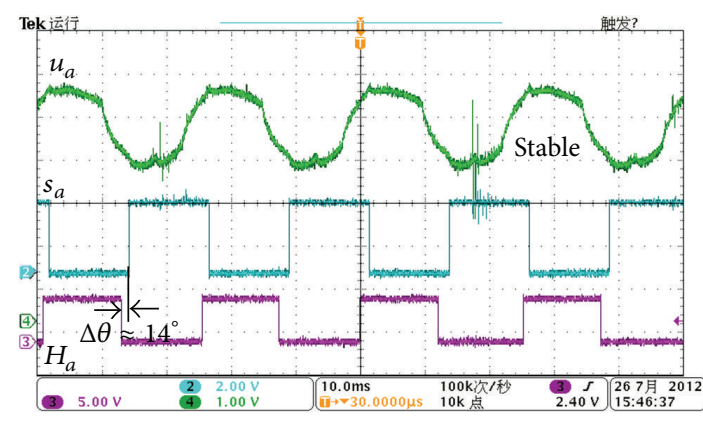

Figure 7: Curves of retarded commutation $\left(\Delta \theta \approx 14^{\circ}\right)$.

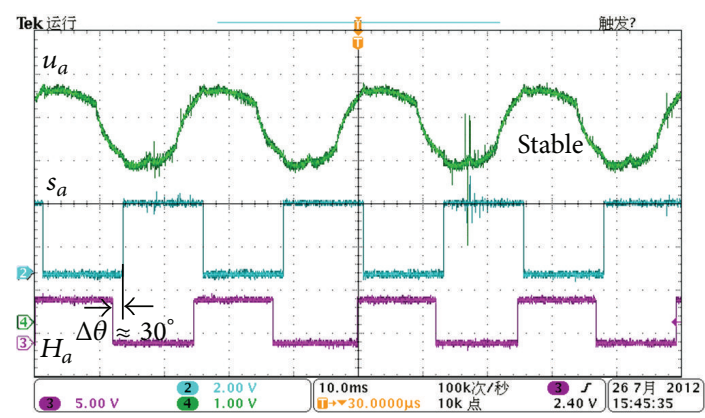

Figure 8: Curves of retarded commutation $\left(\Delta \theta \approx 30^{\circ}\right)$.

for the surface-mounted BLDCM, and then the stability of the open-loop control mode is analyzed and verified by using Lyapunov indirect method. The main conclusions are as follows.

(1) When the commutation instant is advanced, that is, the stator current leads the back EMF, the motor drive system is instable and BLDCM can not operate stably in the open-loop mode.

(2) When the commutation instant is accurate, that is, the stator current is in phase with the back EMF, the motor drive system is marginally stable. However, BLDCM can not operate stably in the open-loop mode yet since there are many kinds of inevitable disturbances in the motor system.

(3) When the commutation instant is retarded, that is, the stator current lags behind the back EMF, the motor drive system is asymptotically stable and BLDCM can operate stably in the open-loop mode.

Based on the results of this paper, further analyses of stability for the open-loop mode can be carried out taking the load and frequency into account. In addition, it is also important to design an effective control strategy for the openloop mode according to the stability results in BLDCMs.

\section{Conflict of Interests}

The authors declare that there is no conflict of interests regarding the publication of this paper. 


\section{Acknowledgment}

The work was partially supported by The National Natural Science Foundation of China (10772011).

\section{References}

[1] K. T. Chau, C. C. Chan, and C. Liu, "Overview of permanentmagnet brushless drives for electric and hybrid electric vehicles," IEEE Transactions on Industrial Electronics, vol. 55, no. 6, pp. 2246-2257, 2008.

[2] P. P. Acarnley and J. F. Watson, "Review of position-sensorless operation of brushless permanent-magnet machines," IEEE Transactions on Industrial Electronics, vol. 53, no. 2, pp. 352-362, 2006.

[3] D. F. Wang, J. Qi, C. Zhu, J. G. Liao, and Y. C. Yuan, "Strategy of starting sensorless BLDCM with inductance method and EMF integration," Mathematical Problems in Engineering, vol. 2013, Article ID 146058, 8 pages, 2013.

[4] J. X. Shen and K. J. Tseng, "Analyses and compensation of rotor position detection error in sensorless PM brushless dc motor drives," IEEE Transactions on Energy Conversion, vol. 18, no. 1, pp. 87-93, 2003.

[5] J. X. Shen and S. Iwasaki, "Sensorless control of ultrahigh-speed PM brushless motor using PLL and third harmonic back EMF," IEEE Transactions on Industrial Electronics, vol. 53, no. 2, pp. 421-428, 2006.

[6] G. C. Verghese, J. H. Lang, and L. F. Casey, "Analysis of instability in electrical machines," IEEE Transactions on Industry Applications, vol. 22, no. 5, pp. 853-864, 1986.

[7] I. E. D. Pickup and A. P. Russell, "Dynamic instability in permanent-magnet synchronous and stepping motors," IEE Proceedings B: Electric Power Applications, vol. 134, no. 2, pp. 91100, 1987.

[8] I. E. D. Pickup and A. P. Russell, "Analysis of frequencyand amplitude-modulation in the stabilisation of permanentmagnet synchronous/stepping motors," IEE Proceedings B: Electric Power Applications, vol. 139, no. 4, pp. 336-346, 1992.

[9] P. D. Chandana Perera, F. Blaabjerg, J. K. Pedersen, and P. Thøgersen, "A sensorless, stable V/f control method for permanent-magnet synchronous motor drives," IEEE Transactions on Industry Applications, vol. 39, no. 3, pp. 783-791, 2003.

[10] N. Matsui, "Sensorless PM brushless DC motor drives," IEEE Transactions on Industrial Electronics, vol. 43, no. 2, pp. 300308, 1996.

[11] H.-C. Chen and C.-M. Liaw, "Current-mode control for sensorless BDCM drive with intelligent commutation tuning," IEEE Transactions on Power Electronics, vol. 17, no. 5, pp. 747-756, 2002.

[12] T. W. Chun, Q. V. Tran, H. H. Lee, and H. G. Kim, "Sensorless control of BLDC motor drive for an automotive fuel pump using a hysteresis comparator," IEEE Transactions on Power Electronics, vol. 29, no. 3, pp. 1382-1391, 2014.

[13] G. H. Jang, J. H. Park, and J. H. Chang, "Position detection and start-up algorithm of a rotor in a sensorless BLDC motor utilising inductance variation," IEE Proceedings: Electric Power Applications, vol. 149, no. 2, pp. 137-142, 2002.

[14] W. J. Lee and S. K. Sul, "A new starting method of BLDC motors without position sensor," IEEE Transactions on Industry Applications, vol. 42, no. 6, pp. 1532-1538, 2006.
[15] B. Asaei and A. Rostami, "A novel starting method for BLDC motors without the position sensors," Energy Conversion and Management, vol. 50, no. 2, pp. 337-343, 2009.

[16] P. Damodharan, R. Sandeep, and K. Vasudevan, "Simple position sensorless starting method for brushless DC motor," IET Electric Power Applications, vol. 2, no. 1, pp. 49-55, 2008.

[17] K. W. Lee, D. K. Kim, B. T. Kim, and B. I. Kwon, "A novel starting method of the surface permanent-magnet BLDC motors without position sensor for reciprocating compressor," IEEE Transactions on Industry Applications, vol. 44, no. 1, pp. 85-92, 2008.

[18] S.-K. Chung, H.-S. Kim, C.-G. Kim, and M.-J. Youn, "A new instantaneous torque control of PM synchronous motor for high-performance direct-drive applications," IEEE Transactions on Power Electronics, vol. 13, no. 3, pp. 388-400, 1998.

[19] Y. Liu, Z. Q. Zhu, and D. Howe, "Direct torque control of brushless DC drives with reduced torque ripple," IEEE Transactions on Industry Applications, vol. 41, no. 2, pp. 599-608, 2005.

[20] K. Tabarraee, J. Iyer, H. Atighechi, and J. Jatskevich, "Dynamic average-value modeling of $120^{\circ} \mathrm{VSI}$-commutated brushless DC motors with trapezoidal back EMF," IEEE Transactions on Energy Conversion, vol. 27, no. 2, pp. 296-307, 2012.

[21] W. Zhang, J. Li, and M. Chen, "Global exponential stability and existence of periodic solutions for delayed reaction-diffusion BAM neural networks with Dirichlet boundary conditions," Boundary Value Problems, vol. 2013, article 105, 2013.

[22] W. Zhang, J. Li, and N. Shi, "Stability analysis for stochastic Markovian jump reaction-diffusion neural networks with partially known transition probabilities and mixed time delays," Discrete Dynamics in Nature and Society, vol. 2012, Article ID 524187, 17 pages, 2012.

[23] W. Zhang and J. Li, "Global exponential stability of reactiondiffusion neural networks with discrete and distributed timevarying delays," Chinese Physics B, vol. 20, no. 3, Article ID 030701, 2011.

[24] W. Zhang and J. Li, "Global exponential synchronization of delayed BAM neural networks with reaction-diffusion terms and the Neumann boundary conditions," Boundary Value Problems, vol. 2012, article 2, 2012.

[25] W. Zhang, J. Li, and M. Chen, "Dynamical behaviors of impulsive stochastic reaction-diffusion neural networks with mixed time delays," Abstract and Applied Analysis, vol. 2012, Article ID 236562, 21 pages, 2012.

[26] W. Zhang, J. Li, and M. Chen, "Adaptive synchronization of the stochastic delayed RDNNs with unknown time-varying parameters," Advances in Difference Equations, vol. 2013, article 253, 2013.

[27] J. Li, W. Zhang, and M. Chen, "Synchronization of delayed reaction-diffusion neural networks via an adaptive learning control approach," Computers \& Mathematics with Applications, vol. 65, no. 11, pp. 1775-1785, 2013. 


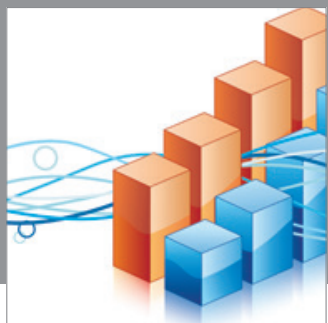

Advances in

Operations Research

mansans

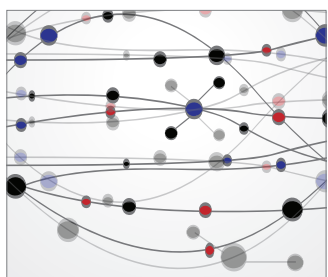

The Scientific World Journal
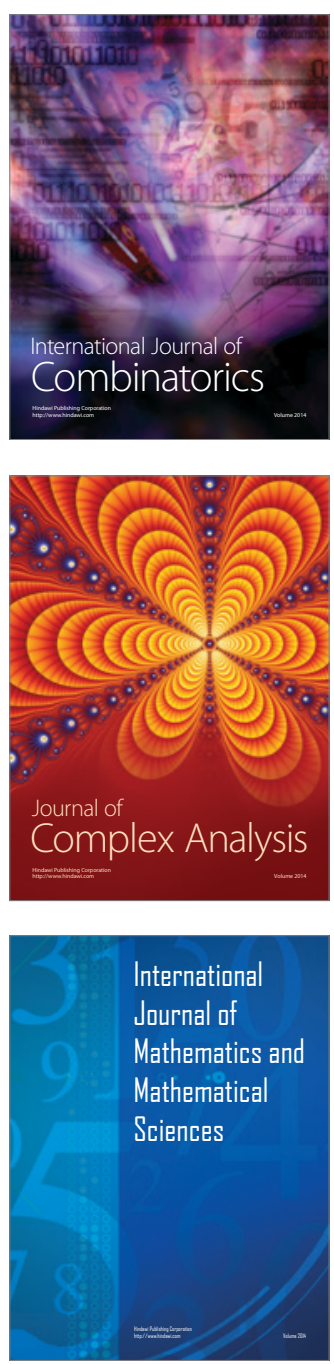
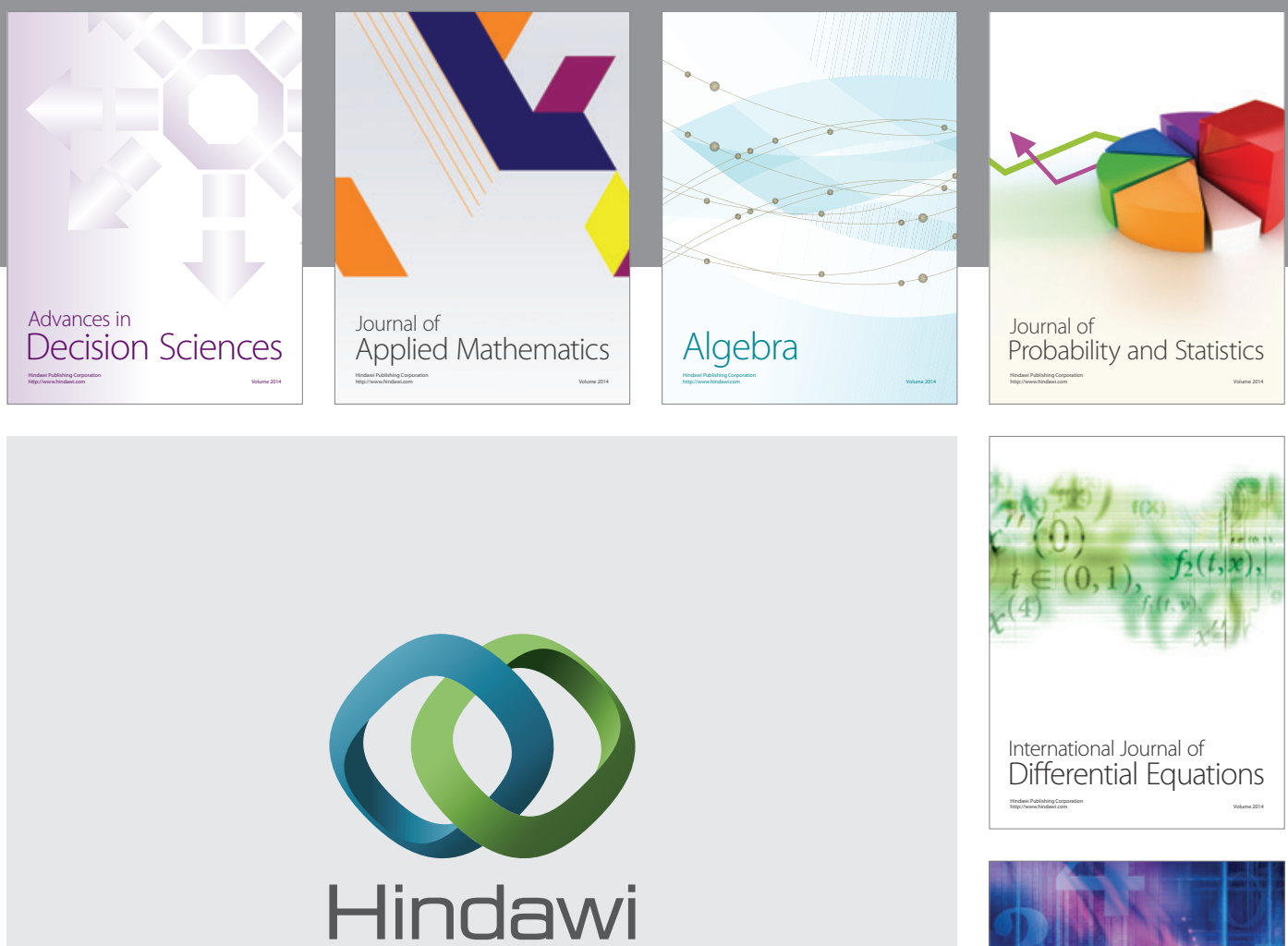

Submit your manuscripts at http://www.hindawi.com
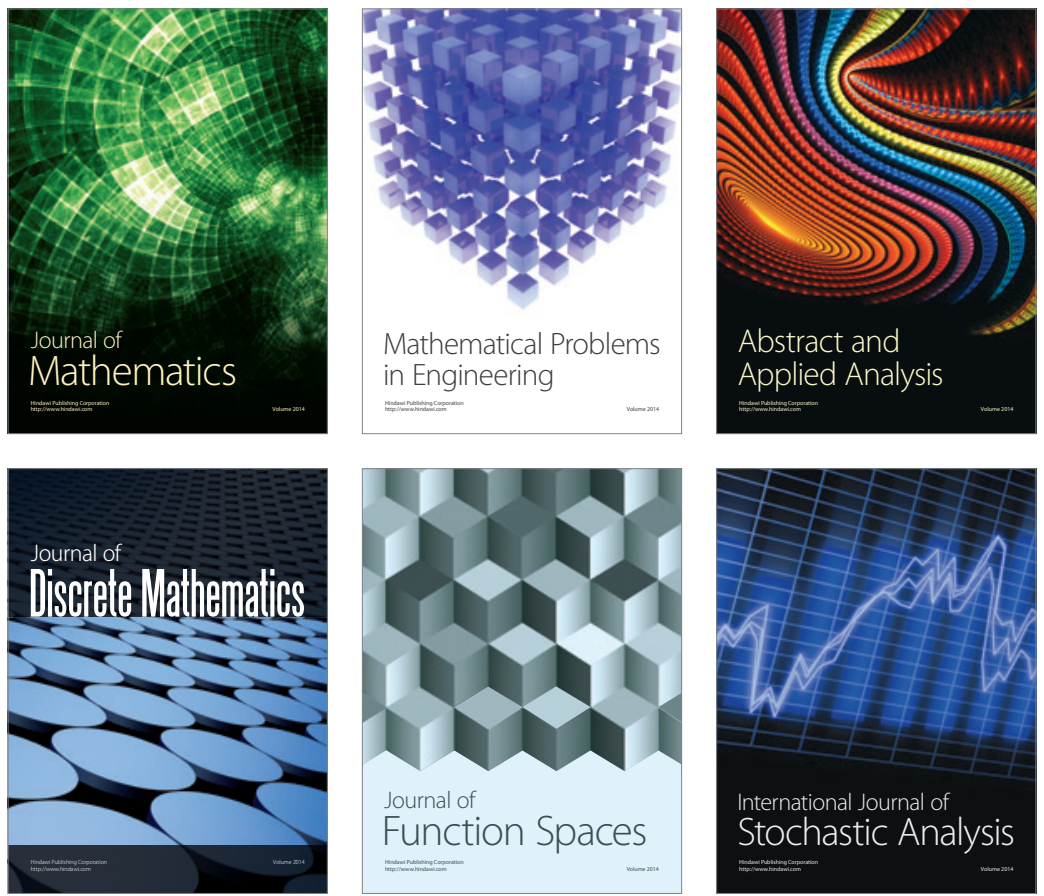

Journal of

Function Spaces

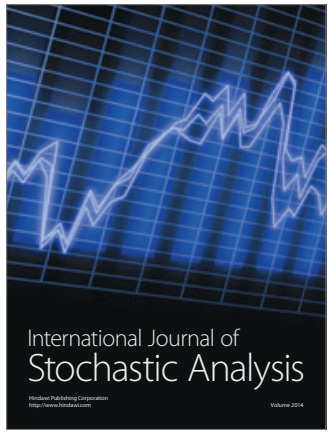

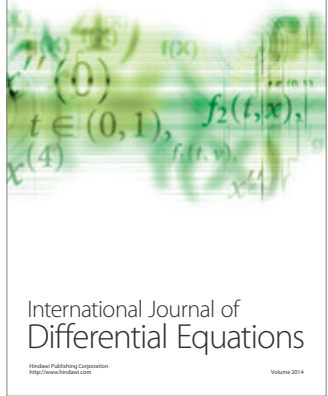
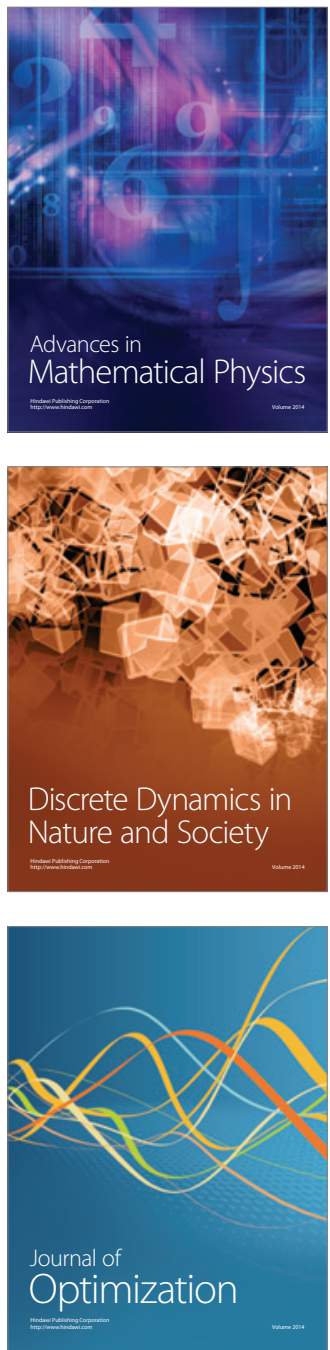